\title{
A Good Numerical Method for the Solution of Generalized Regularized Long Wave Equation
}

\author{
Fuchang Zheng ${ }^{1}$, Shuhong Bao ${ }^{1}$, Yulan Wang ${ }^{1}$, Shuguang $\mathrm{Li}^{1} \&$ Zhiyuan $\mathrm{Li}^{1}$ \\ ${ }^{1}$ College of Science, Inner Mongolia University of Technology, Hohhot, China \\ Correspondence: Shuhong Bao, College of Science, Inner Mongolia University of Technology, Hohhot, China. \\ E-mail: bshnei@163.com
}

Received: May 29, 2017

doi:10.5539/mas.v11n6p72
Accepted: April 7, 2017

Online Published: April 17, 2017

URL: https://doi.org/10.5539/mas.v11n6p72

\begin{abstract}
The generalized regularized long wave equation is very important that can be applied in the field of physics, science and technology. Some authors have put forward many different numerical method, but the precision is not enough high. In this paper, we will illustrate the high-precision numerical method to solve the generalized regularized long wave equation. Three numerical examples are studied to demonstrate the accuracy of the present method. Results obtained by our method indicate new algorithm has the following advantages: small computational work, fast convergence speed and high precision.
\end{abstract}

Keywords: generalized regularized long wave equation, high precision numerical method, partial differential equation

\section{Introduction}

The nonlinear wave is one of the most important scientific research areas. The generalized regularized long wave (GRLW) equation can be used to describe nonlinear dispersive waves and the phenomena of weak nonlinearity and dispersion waves, including nonlinear transverse waves in shallow water, ion-acoustic and magneto hydrodynamic waves in plasma, phonon packets in nonlinear crystals and a large number of physical phenomena. The GRLW equation can be written as:

$$
\frac{\partial u}{\partial t}+\alpha \frac{\partial u}{\partial x}+\beta u \frac{\partial u}{\partial x}-\mu \frac{\partial^{2} u}{\partial x^{2}}-\delta \frac{\partial^{3} u}{\partial x^{2} \partial t}=0 \quad \Omega \in[a, b] \times[0, T]
$$

The regularized long wave (RLW) equation for $\alpha=1, \beta \neq 0, \delta \neq 0$, and $\mu=0$. where $\alpha, \beta, \mu$ and $\delta$ are nonnegative constants, $\beta$ and $\delta$ are the behavior of an undular bore, the nonlinear term $\beta \mu \frac{\partial u}{\partial x}$ causes the wave form steep, $\delta \frac{\partial^{3} u}{\partial x^{2} \partial t}$ is the dispersion effect term that makes the wave form spread, the solitons appear as a result of the balance between this weak nonlinearity and dispersion, $t$ is the time and $x$ is the space coordinate. It is well known that solving the GRLW equation have many methods. Some of the previous works on the GRLW equation include an implicit second-order accurate and stable energy preserving finite difference method based on the use of central difference equations for the time and space derivatives (Lu-Ming, 2005), a method of lines based on the discretization of the spatial derivatives by means of Fourier pseudo-spectral approximations (Durn, 2002), a Fourier spectral method for the initial value problem of the GRLWequation (Ben-Yu, 1988), and a linearized implicit pseudo-spectral method (Djidjeli, 2003). Moreover numerical techniques such as finite difference methods (Ramos, J. I., 2006), a spectral method (Hammada, 2016), finite element methods based on the least square principle (Fu-Zheng, 2015), finite element methods based on Galerkin and collocation principles (Noureddine, 2013), the Petrov-Galerkin method (Thoudam, 2012), the radial basis function collocation method (Siraj-ul-Islam, 2009), the Sinc-collocation method (Reza, 2010), the collocation method with quintic B-splines (Battal, 2016) and the cubic B-spline finite element method (Khalifa, 2008) have been devised for finding numerical solutions of special kinds of GRLW equations. As we know, the barycentric interpolation collocation method already applied to the integral calculus, linear equation and ordinary differential equation very well, but few people to applied to nonlinear partial differential equation. In the following, we extending the barycentric 
interpolation collocation method to solve the GRLW equation.

In this study, a new algorithm for solving the GRLW equation in the meshless space is proposed. The advantages of the approach show in the following facts. The paper is organized as follows. Section 2 is recommend the barycentric interpolation collocation method.Through numerical examples to illustrate the advantages in section 3 . The last section is a brief conclusion.

\section{Barycentric Interpolation Collocation Method}

\subsection{The Partial Differential Matrix of Barycentric Interpolation}

In this section, we illustrate the partial differential matrix of barycentric interpolation. We consider a regular rectangle region $\Omega=[a, b] \times[0, T]$. It divided into $M$ different nodes: $a=x_{1}<x_{2}<\cdots<x_{M}=b$ in the interval $[a, b]$, and also divided into $N$ different nodes in the interval $[0, T]: 0=t_{1}<t_{2}<\cdots<t_{N}=T$. In the region $\Omega$, the nodes generate tensor type nodes, that is: $\left\{\left(x_{i}, t_{j}\right), i=1,2, \cdots, M ; j=1,2, \cdots, N\right\}$.

The functional value $u(x, t)$ at nodes $\left(x_{i}, t_{j}\right)$ is defined as:

$$
u_{i j}=u\left(x_{i}, t_{j}\right) \quad i=1,2, \cdots, M, j=1,2, \cdots, N
$$

The barycentric interpolation of $u(x, t)$ at nodes $\left(x_{i}, t_{j}\right)$ can be written as:

$$
u(x, t)=\sum_{i=1}^{M} \sum_{j=1}^{N} \xi_{i}(x) \eta_{j}(t) u_{i j}
$$

Here, $\xi_{i}(x), \eta_{j}(t)$ are respectively barycentric interpolation basis function of $u(x, t)$ on the interval $[a, b]$ nodes and the interval $[0, T]$ nodes.

Use formula (3), the $p+q$ order partial derivative of function $u(x, t)$ at nodes can be expressed as:

$$
\frac{\partial^{p+q} u}{\partial x^{p} \partial t^{q}}=\sum_{i=1}^{M} \sum_{j=1}^{N} \xi_{i}^{(p)}(x) \eta_{j}^{(q)}(t) u_{i j} \quad p, q=0,1,2, \cdots,
$$

and the functional values of partial derivative at nodes $\left(x_{i}, t_{j}\right)$ are defined as:

$$
u^{(p, q)}\left(x_{l}, t_{k}\right)=\frac{\partial^{p+q} u\left(x_{l}, t_{k}\right)}{\partial x^{p} \partial t^{q}}=\sum_{i=1}^{M} \sum_{j=1}^{N} \xi_{i}^{(p)}\left(x_{l}\right) \eta_{j}^{(q)}\left(t_{k}\right) u_{i j} \quad l=1,2, \cdots, M ; k=1,2, \cdots, N
$$

Let $x_{0}=\left[x_{1}, x_{2}, \cdots, x_{M}\right]^{T}, t_{0}=\left[t_{1}, t_{2}, \cdots, t_{N}\right]^{T}$ be respectively defineded as the column vectors of nodes at $x, t$ axis, and the matrixes $X, T$ composed of tensor type node coordinates are respectively defined as:

$$
X=\left[x_{0}^{T}, x_{0}^{T}, \cdots, x_{0}^{T}\right]^{T} \quad T=\left[t_{0}, t_{0}, \cdots, t_{0}\right]
$$

Let the matrix $X, T$ by columns stretch into a $N \times M$ dimensional column vector $x$, t, that is:

$$
x=\left[X_{1}, X_{2}, \cdots, X_{M \times N}\right]^{T} \quad t=\left[T_{1}, T_{2}, \cdots, T_{M \times N}\right]^{T}
$$

The relationship between components of vector $x, t$ and vector $x_{0}, t_{0}$ exist the following :

$$
X_{q}=X_{(i-1) N+j}=x_{i}, T_{q}=T_{(i-1) N+j}=t_{j} \quad i=1,2, \cdots, M ; j=1,2, \cdots, N ; q=1,2, \cdots, M \times N
$$

To facilitate, let

$$
\begin{gathered}
u=\left[u_{1}, u_{2}, \cdots, u_{M \times N}\right]^{T} \quad u^{(p, q)}=\left[u_{1}^{(p, q)}, u_{2}^{(p, q)}, \cdots, u_{M \times N}^{(p, q)}\right]^{T} \\
u_{l}=u\left(X_{l}, T_{l}\right), u_{l}^{(p, q)}=u^{(p, q)}\left(X_{l}, T_{l}\right) \quad l=1,2, \cdots, M \times N
\end{gathered}
$$

So,formula (8) can be written in following matrix form:

$$
u^{(p, q)}=D^{(p, q)} u
$$

In the above formula, $D^{(p, q)}=C^{(p)} \otimes D^{(q)}$ is the Kronecker product of matrix $C^{(p)}$ and $D^{(q)}$, and it is also called $p+q$ order partial differential matrix at nodes $\left\{\left(x_{i}, t_{j}\right), i=1,2, \cdots, M ; j=1,2, \cdots, N\right\} . \quad C^{(p)}$ and $D^{(q)}$ are respectively called $p$ order partial differential matrix and $q$ order partial differential matrix. Denote:

$$
C^{(0)}=I_{M} \quad D^{(0)}=I_{N}
$$


where $I_{M}$ and $I_{N}$ are respectively $M$ order unit matrix and $N$ order unit matrix.

The partial differential matrix has the following relations:

$$
D^{(p, 0)}=\left(D^{(1,0)}\right)^{p}, \quad D^{(0, q)}=\left(D^{(0,1)}\right)^{q}
$$

Use the barycentric interpolation collocation method to solve the partial differential equation, there are several types of nodes to choose. Such as: equidistant nodes, Chebyshev nodes, Legendre nodes and so on.

The Chebyshev nodes calculation formula is:

$$
x_{i}=-\cos \left(\frac{i-1}{M-1} \pi\right), t_{j}=-\cos \left(\frac{j-1}{N-1} \pi\right), \quad i=1,2, \cdots, M ; j=1,2, \cdots, N
$$

This paper that we use the Chebyshev nodes, which can improve the accuracy effectively.

\subsection{Direct Linearized Iterative Method}

Next, we put the nonlinear equations transformed into linear equations and introduce a direct linearized iterative method for solving nonlinear equations.

The nonlinear partial differential equation $\mathcal{D} u(x, t)=f(x, t)$ can divided into two parts, which are linear term and nonlinear term:

$$
\mathcal{L} u(x, t)+\mathcal{N} u(x, t)=f(x, t)
$$

in the formula, $\mathcal{L}$ is linear differential operator and $\mathcal{N}$ is nonlinear differential operator.

Take initial hypothesis function $u_{0}(x, t)$ which has been given into formula (11), we obtain:

$$
\mathcal{L} u(x, t)+\mathcal{N} u_{0}(x, t)=f(x, t)
$$

Therefore, the formula(12)is transformed into a linear differential equation. We can get a new function $u_{1}(x, t)$ after solving formula (12), and through the iteration we can obtain:

$$
\mathcal{L} u_{n}(x, t)+\mathcal{N} u_{n-1}(x, t)=f(x, t)
$$

A control precision $\varepsilon$ is given in the course of the actual calculation, if $\left|u_{n}(x)-u_{n-1}(x)\right| \leqslant \varepsilon$, then the iteration stops, the approximate solution of the nonlinear problem is obtained. This process is referred to as the direct linearized iteration method.

\subsection{The Barycentric Interpolation Collocation Formula of the Generalized Regularized Long Wave Equation}

Think about function (1)

$$
\frac{\partial u}{\partial t}+\alpha \frac{\partial u}{\partial x}+\beta u \frac{\partial u}{\partial x}-\mu \frac{\partial^{2} u}{\partial x^{2}}-\delta \frac{\partial^{3} u}{\partial x^{2} \partial t}=0
$$

where $\alpha=1, \beta \neq 0, \delta \neq 0$ and $\mu=0$. So the function can be written as

$$
\frac{\partial u}{\partial t}+\frac{\partial u}{\partial x}+\beta u \frac{\partial u}{\partial x}-\delta \frac{\partial^{3} u}{\partial x^{2} \partial t}=0
$$

the nonlinear term is $\beta u \frac{\partial u}{\partial x}$. Take initial hypothesis function $u_{0}(x, t)$ which has been given into formula (15), we obtain:

$$
\frac{\partial u}{\partial t}+\frac{\partial u}{\partial x}+\beta u_{0} \frac{\partial u}{\partial x}-\delta \frac{\partial^{3} u}{\partial x^{2} \partial t}=0
$$

Therefore,the formula(16)is transformed into a linear differential equation. We can get a new function $u_{1}(x, t)$ after solving formula (16), and we can get a linear iterative scheme

$$
\frac{\partial u_{n}}{\partial t}+\frac{\partial u_{n}}{\partial x}+\beta u_{n-1} \frac{\partial u_{n}}{\partial x}-\delta \frac{\partial^{3} u_{n}}{\partial x^{2} \partial t}=0
$$

A control precision $\varepsilon$ is given in the course of the actual calculation, if $\left|u_{n}(x)-u_{n-1}(x)\right| \leqslant \varepsilon$, then the iteration stops, the approximate solution of the nonlinear problem is obtained. This process is referred to as the direct linearized iteration method. 


\subsection{The Convergence Analysis of the Generalized Regularized Long Wave Equation}

Let $E$ be $\mathcal{L} u+\mathcal{N} U=f$, let $E_{n}$ be $T_{n} U=\mathcal{L} u+\mathcal{N} U_{n}=f$.

$\left\{E_{n}\right\}$ is a sequence of the linear partial differential equation, then

$$
T_{n} U=f
$$

On account of knowledge of the topology, to the continuous deformation $E$, then

$$
\mathcal{L} u+\mathcal{N} U=f
$$

Assumption the genuine solution is $U^{* *}$, it can approximate representation linear equation $T U=f$.

Assumption the solution is $U^{*}$, then $U^{* *}$ and $U^{*}$ are arbitrarily close. And the continuous linear operator $T_{n}$ converge to the continuous linear operator $T$.

$$
T_{n} U_{n}=f
$$

then $T U_{n}$ converge to $f$, then $T\left(U_{n}-U^{*}\right)$ converge to 0 , therefore $U_{n}$ converge to $U^{*}$, thereby $U_{n}$ converge to $U^{* *}$. Assumption the nonlinear operator $N$ is differentiable of Frechet.

Then (1) $\mathcal{N}\left(x_{0}+h\right)$ converge to $\mathcal{N}\left(x_{0}\right),(h$ converge to 0$)$.

(2) exist bounded linear operator $T_{0}$,

$$
\mathcal{N}\left(x_{0}+h\right)=\mathcal{N}\left(x_{0}\right)+T_{0} h+w\left(x_{0}, h\right)
$$

thereinto $\frac{\left\|w\left(x_{0}, h\right)\right\|}{\|h\|}$ converge to $0,(\|h\|$ converge to 0$)$.

So $\mathcal{N}$ is linearization.

\section{Numerical Experiment}

In this section, three numerical examples are studied to demonstrate the advantage of the present method.

Example $1^{[14-16]}$ Considering following nonlinear RLW equation with initial condition and boundary conditions.

$$
\frac{\partial u}{\partial t}+\alpha \frac{\partial u}{\partial x}+\beta u \frac{\partial u}{\partial x}-\delta \frac{\partial^{3} u}{\partial x^{2} \partial t}=0 \quad(x, t) \in(a, b) \times(0, T)
$$

The RLW equation has three conservation quantities corresponding to mass, momentum and energy:

$$
\begin{aligned}
& I_{1}=\int_{a}^{b} u \mathrm{~d} x \\
& I_{2}=\int_{a}^{b}\left(u^{2}+\delta\left(\frac{\partial u}{\partial x}\right)^{2}\right) \mathrm{d} x \\
& I_{3}=\int_{a}^{b}\left(u^{3}+3 u^{2}\right) \mathrm{d} x
\end{aligned}
$$

The RLW equation has an analytical solution of the form is

$$
u(x, t)=3 c \operatorname{sech}^{2}\left(k\left[x-x_{0}-v t\right]\right)
$$

where $v=1+\beta c$ is the wave velocity, $k=\frac{1}{2} \sqrt{\frac{\beta c}{\delta v}}$ and $c$ is a constant. This solution represents the motion of a single solitary wave with amplitude $3 \mathrm{c}$ and initially centered at $x_{0}=0$ and $\alpha=\beta=\delta=1$.

The initial condition is taken as

$$
u(x, 0)=3 c \operatorname{sech}^{2}\left(k\left[x-x_{0}\right]\right)
$$

The numerical experiments are done in the region $-40 \leq x \leq 60, T=20$ with parameters $c=0.1, M=150, N=$ 120 that are presented in Table 1.Fig 1. With the increase of the points, accuracy is higher and highter that are presented in Table 2.Fig 2.

The numerical experiments are done in the region $-80 \leq x \leq 120, T=20$ with parameters $c=0.03, M=150, N=$ 120 that are presented in Table 3.Fig 3.

Example $2^{[17]}$ Considering following nonlinear RLW equation with initial condition and boundary conditions.

$$
\frac{\partial u}{\partial t}+\frac{\partial u}{\partial x}+u \frac{\partial u}{\partial x}-\frac{\partial^{3} u}{\partial x^{2} \partial t}=0 \quad(x, t) \in(a, b) \times(0, T)
$$

The RLW equation has an analytical solution of the form is

$$
u(x, t)=A \operatorname{sech}^{2}(k x-w t+\delta)
$$


The initial condition is taken as

$$
u(x, 0)=\operatorname{sech}^{2}\left(\frac{x}{4}\right)
$$

where

$$
A=\frac{3 a^{2}}{1-a^{2}} \quad k=\frac{a}{2} \quad w=\frac{a}{2\left(1-a^{2}\right)}
$$

We give the numerical solution in the region $-50 \leq x \leq 50, a=0.5, x_{0}=10, M=200, N=100, T=1$ that are presented in Table 4.Fig 4.

Example $3^{[6,18]}$ Considering following nonlinear RLW equation with initial condition and boundary conditions.

$$
\frac{\partial u}{\partial t}+\frac{\partial u}{\partial x}+q \frac{\partial u^{q+1}}{\partial x}-\mu \frac{\partial^{3} u}{\partial x^{2} \partial t}=0 \quad(x, t) \in(a, b) \times(0, T)
$$

where the numerical experiments are done in the region $0 \leq x \leq 100, T=10, q=1, \mu=1$.

Single solitary wave: the RLW equation has an analytical solution of the form is

$$
u(x, t)=\left[\frac{v(q+2)}{2 q} \operatorname{sech}^{2}\left(\frac{q}{2} \sqrt{\frac{v}{\mu(v+1)}}\left(x-x_{0}-(v+1) t\right)\right)\right]^{\frac{1}{q}}
$$

We consider the initial condition of given by

$$
u(x, 0)=\left[\frac{v(q+2)}{2 q} \operatorname{sech}^{2}\left(\frac{q}{2} \sqrt{\frac{v}{\mu(v+1)}}\left(x-x_{0}\right)\right)\right]^{\frac{1}{q}}
$$

where amplitude (A) and the inverse width (B) of the RLW solution are respectively given by $A=\left[\frac{v(q+2)}{2 q}\right]^{\frac{1}{q}}$ and $B=\frac{q}{2} \sqrt{\frac{v}{\mu(v+1)}}$ and $v=0.1, x_{0}=40, M=150, N=100, T=10$ that are presented in Table 5.Fig 5.

Interaction of two solitary waves: the RLW equation has an analytical solution of the form is

$$
u(x, t)=\sum_{j=1}^{2}\left[\frac{v_{j}(q+2)}{2 q} \operatorname{sech}^{2}\left(\frac{q}{2} \sqrt{\frac{v_{j}}{\mu\left(v_{j}+1\right)}}\left(x_{j}-x_{0 j}-\left(v_{j}+1\right) t\right)\right)\right]^{\frac{1}{q}}
$$

Here, we take the initial condition given by the form

$$
u(x, 0)=\sum_{j=1}^{2}\left[\frac{v_{j}(q+2)}{2 q} \operatorname{sech}^{2}\left(\frac{q}{2} \sqrt{\frac{v_{j}}{\mu\left(v_{j}+1\right)}}\left(x_{j}-x_{0 j}\right)\right)\right]^{\frac{1}{q}}
$$

where amplitude $A_{j}$ and the inverse width $B_{j}$ of the RLW solution are respectively given by $A_{j}=\left[\frac{v_{j}(q+2)}{2 q}\right]^{\frac{1}{q}}$ and $B_{j}=\frac{q}{2} \sqrt{\frac{v_{j}}{\mu\left(v_{j}+1\right)}}$ and $v_{1}=0.2, v_{2}=0.1, x_{01}=15, x_{02}=35, M=100, N=40, T=10$ that are presented in Table 6.Fig 6 .

Table 1. Comparison of $L_{2}$ and $L_{\infty}$ for Example 1

\begin{tabular}{lcccccc}
\hline t & Present method & Method in[14] & Method in[15] & Present method & Method in[14] & Method in[15] \\
& $L_{2}$ & $L_{2}$ & $L_{2}$ & $L_{\infty}$ & $L_{\infty}$ & $L_{\infty}$ \\
\hline 4 & $1.0547 \mathrm{E}-11$ & $1.1766 \mathrm{E}-04$ & $7.8155 \mathrm{E}-05$ & $5.1947 \mathrm{E}-13$ & $5.6222 \mathrm{E}-05$ & $3.4877 \mathrm{E}-05$ \\
8 & $1.4972 \mathrm{E}-11$ & $2.3108 \mathrm{E}-04$ & $1.2283 \mathrm{E}-04$ & $9.5152 \mathrm{E}-13$ & $1.0880 \mathrm{E}-04$ & $5.0934 \mathrm{E}-05$ \\
12 & $7.5147 \mathrm{E}-12$ & $3.3746 \mathrm{E}-04$ & $1.7123 \mathrm{E}-04$ & $5.9144 \mathrm{E}-13$ & $1.5442 \mathrm{E}-04$ & $6.5477 \mathrm{E}-05$ \\
16 & $5.6144 \mathrm{E}-12$ & $4.3784 \mathrm{E}-04$ & $2.1954 \mathrm{E}-04$ & $3.9707 \mathrm{E}-13$ & $1.9377 \mathrm{E}-04$ & $7.8911 \mathrm{E}-05$ \\
20 & $9.1130 \mathrm{E}-12$ & $5.3223 \mathrm{E}-04$ & $2.6685 \mathrm{E}-04$ & $1.0376 \mathrm{E}-12$ & $2.2722 \mathrm{E}-04$ & $9.1465 \mathrm{E}-05$ \\
\hline
\end{tabular}


Table 2. The numerical results of Example 1

\begin{tabular}{lccc}
\hline $\mathrm{n}$ & Absolute errors & Relative errors & Infinite norm \\
\hline 20 & $1.1661 \mathrm{E}-01$ & $7.9394 \mathrm{E}-02$ & $8.4112 \mathrm{E}-03$ \\
40 & $5.6901 \mathrm{E}-03$ & $1.8522 \mathrm{E}-03$ & $6.2929 \mathrm{E}-04$ \\
60 & $1.3896 \mathrm{E}-04$ & $3.2009 \mathrm{E}-05$ & $3.2498 \mathrm{E}-06$ \\
80 & $2.6422 \mathrm{E}-06$ & $4.5742 \mathrm{E}-07$ & $7.4717 \mathrm{E}-08$ \\
100 & $3.9182 \mathrm{E}-08$ & $5.4334 \mathrm{E}-09$ & $9.8226 \mathrm{E}-10$ \\
120 & $6.1834 \mathrm{E}-10$ & $7.1516 \mathrm{E}-11$ & $1.0035 \mathrm{E}-11$ \\
\hline
\end{tabular}

Table 3. Comparison of $L_{\infty}$ for Example 1

\begin{tabular}{lcc}
\hline Method & Time & Infinite norm \\
\hline Present method(N=100) & 4 & $2.2599 \mathrm{E}-07$ \\
Present method(N=100) & 8 & $2.5633 \mathrm{E}-07$ \\
Present method(N=100) & 12 & $2.6558 \mathrm{E}-07$ \\
Present method(N=100) & 16 & $2.8010 \mathrm{E}-07$ \\
Present method(N=100) & 20 & $2.9264 \mathrm{E}-07$ \\
Reproducing kernel space[16] & 20 & $7.1755 \mathrm{E}-04$ \\
Galerkin linear[16] & 20 & $1.9800 \mathrm{E}-04$ \\
Least square cubic[16] & 20 & $1.5661 \mathrm{E}-03$ \\
Galerkin[16] & 20 & $4.3914 \mathrm{E}-04$ \\
Cubic B-spline collocation[16] & 20 & $3.6498 \mathrm{E}-04$ \\
\hline
\end{tabular}

Table 4. Comparison the numerical results for Example 2

\begin{tabular}{cccc}
\hline $\mathrm{t}$ & $\begin{array}{c}\text { Present method } \\
2 \text { norm }\end{array}$ & $\begin{array}{c}\text { Present method } \\
\text { Infinite norm }\end{array}$ & $\begin{array}{c}\text { Method in [17] } \\
\text { Square errors }\end{array}$ \\
\hline 0.2 & $7.37 \mathrm{E}-10$ & $4.03 \mathrm{E}-11$ & $2.26 \mathrm{E}-03$ \\
0.4 & $8.17 \mathrm{E}-10$ & $3.64 \mathrm{E}-11$ & $3.40 \mathrm{E}-03$ \\
0.6 & $1.04 \mathrm{E}-09$ & $3.24 \mathrm{E}-11$ & $4.54 \mathrm{E}-03$ \\
0.8 & $1.06 \mathrm{E}-09$ & $3.64 \mathrm{E}-11$ & $5.68 \mathrm{E}-03$ \\
1.0 & $9.29 \mathrm{E}-10$ & $3.87 \mathrm{E}-11$ & $6.82 \mathrm{E}-03$ \\
\hline
\end{tabular}

Table 5. Comparison of $L_{2}$ and $L_{\infty}$ for Example 3 (Single solitary wave)

\begin{tabular}{ccccc}
\hline $\mathrm{t}$ & $\begin{array}{c}\text { Present method } \\
L_{2}\end{array}$ & $\begin{array}{c}\text { Method in [6] } \\
L_{2}\end{array}$ & $\begin{array}{c}\text { Present method } \\
L_{\infty}\end{array}$ & $\begin{array}{c}\text { Method in [6] } \\
L_{\infty}\end{array}$ \\
\hline 1 & $2.66 \mathrm{E}-11$ & $2.97 \mathrm{E}-04$ & $2.38 \mathrm{E}-12$ & $4.18 \mathrm{E}-04$ \\
2 & $9.87 \mathrm{E}-11$ & $4.60 \mathrm{E}-04$ & $5.79 \mathrm{E}-12$ & $5.31 \mathrm{E}-04$ \\
3 & $1.16 \mathrm{E}-11$ & $4.90 \mathrm{E}-04$ & $1.05 \mathrm{E}-12$ & $5.66 \mathrm{E}-04$ \\
4 & $5.28 \mathrm{E}-12$ & $4.27 \mathrm{E}-04$ & $2.64 \mathrm{E}-13$ & $4.85 \mathrm{E}-04$ \\
5 & $5.23 \mathrm{E}-12$ & $3.23 \mathrm{E}-04$ & $6.29 \mathrm{E}-13$ & $3.37 \mathrm{E}-04$ \\
6 & $2.38 \mathrm{E}-12$ & $3.20 \mathrm{E}-04$ & $1.24 \mathrm{E}-13$ & $3.48 \mathrm{E}-04$ \\
7 & $6.43 \mathrm{E}-12$ & $4.72 \mathrm{E}-04$ & $3.81 \mathrm{E}-13$ & $6.90 \mathrm{E}-04$ \\
8 & $5.76 \mathrm{E}-12$ & $5.77 \mathrm{E}-04$ & $5.89 \mathrm{E}-13$ & $8.55 \mathrm{E}-04$ \\
9 & $1.36 \mathrm{E}-11$ & $5.06 \mathrm{E}-04$ & $1.56 \mathrm{E}-12$ & $6.36 \mathrm{E}-04$ \\
10 & $2.54 \mathrm{E}-12$ & $2.95 \mathrm{E}-04$ & $1.69 \mathrm{E}-13$ & $2.69 \mathrm{E}-04$ \\
\hline
\end{tabular}

Table 6. Comparison of $L_{2}$ and $L_{\infty}$ for Example 3 (Two solitary waves)

\begin{tabular}{lcccc}
\hline $\mathrm{t}$ & $\begin{array}{c}\text { Present method } \\
L_{2}\end{array}$ & $\begin{array}{c}\text { Method in [18] } \\
L_{2}\end{array}$ & $\begin{array}{c}\text { Present method } \\
L_{\infty}\end{array}$ & $\begin{array}{c}\text { Method in [18] } \\
L_{\infty}\end{array}$ \\
\hline 2 & $4.4911 \mathrm{E}-03$ & $4.1696 \mathrm{E}-02$ & $4.6940 \mathrm{E}-04$ & $1.7435 \mathrm{E}-02$ \\
4 & $9.0206 \mathrm{E}-03$ & $3.7089 \mathrm{E}-02$ & $9.9364 \mathrm{E}-04$ & $1.4583 \mathrm{E}-02$ \\
6 & $1.3641 \mathrm{E}-02$ & $3.2837 \mathrm{E}-02$ & $1.5531 \mathrm{E}-03$ & $1.2193 \mathrm{E}-02$ \\
8 & $1.8395 \mathrm{E}-02$ & $2.9251 \mathrm{E}-02$ & $2.2151 \mathrm{E}-03$ & $1.0766 \mathrm{E}-02$ \\
10 & $2.3317 \mathrm{E}-02$ & $2.6704 \mathrm{E}-02$ & $2.9649 \mathrm{E}-03$ & $1.0324 \mathrm{E}-02$ \\
\hline
\end{tabular}



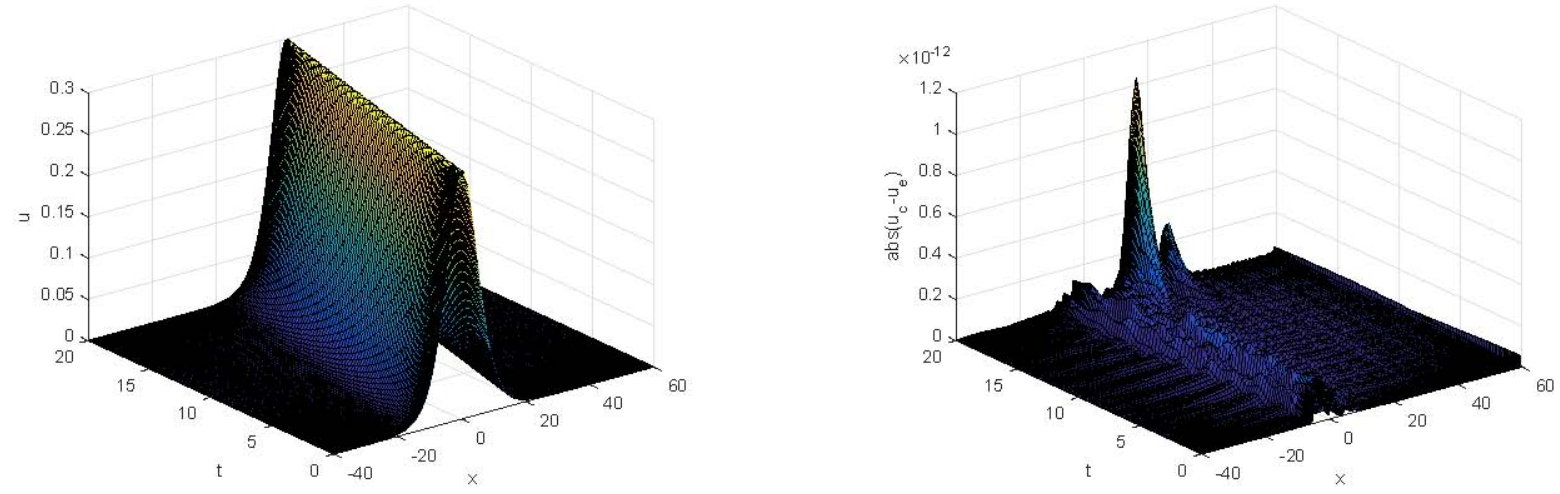

Figure 1. $(-40 \leq x \leq 60)$ : The numerical solution of Example 1. The absolute error of Example 1
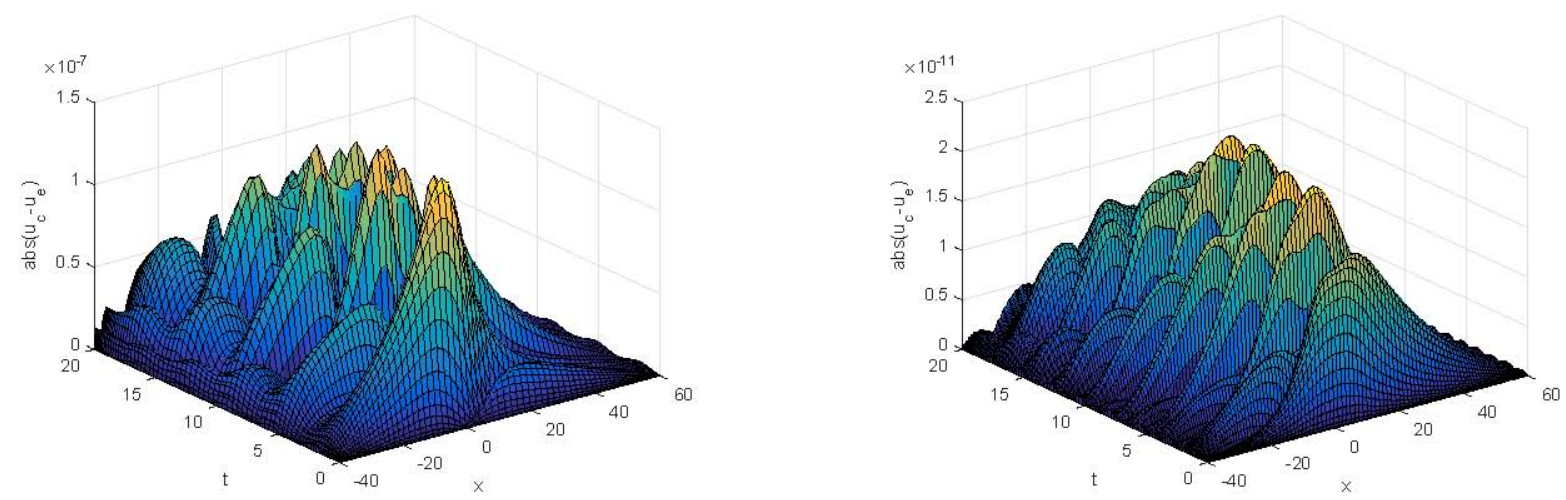

Figure 2. The absolute error of Example 1. $(\mathrm{N}=80)$ The absolute error of Example 1.(N=120)

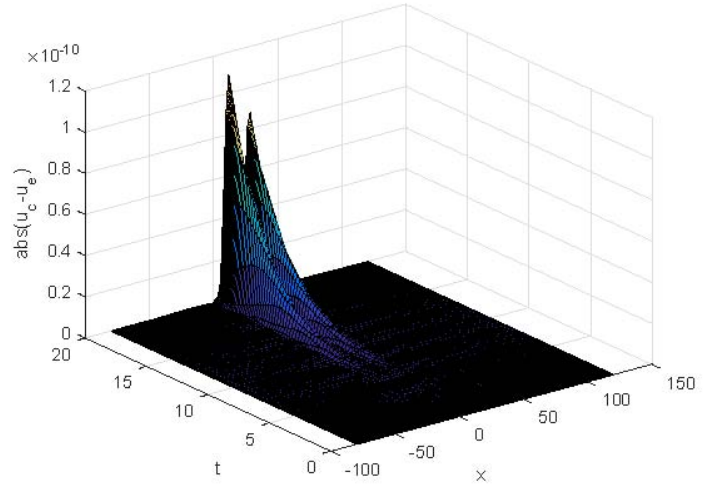

Figure 3. $(-80 \leqslant x \leqslant 120)$ : The absolute error of Example 1 

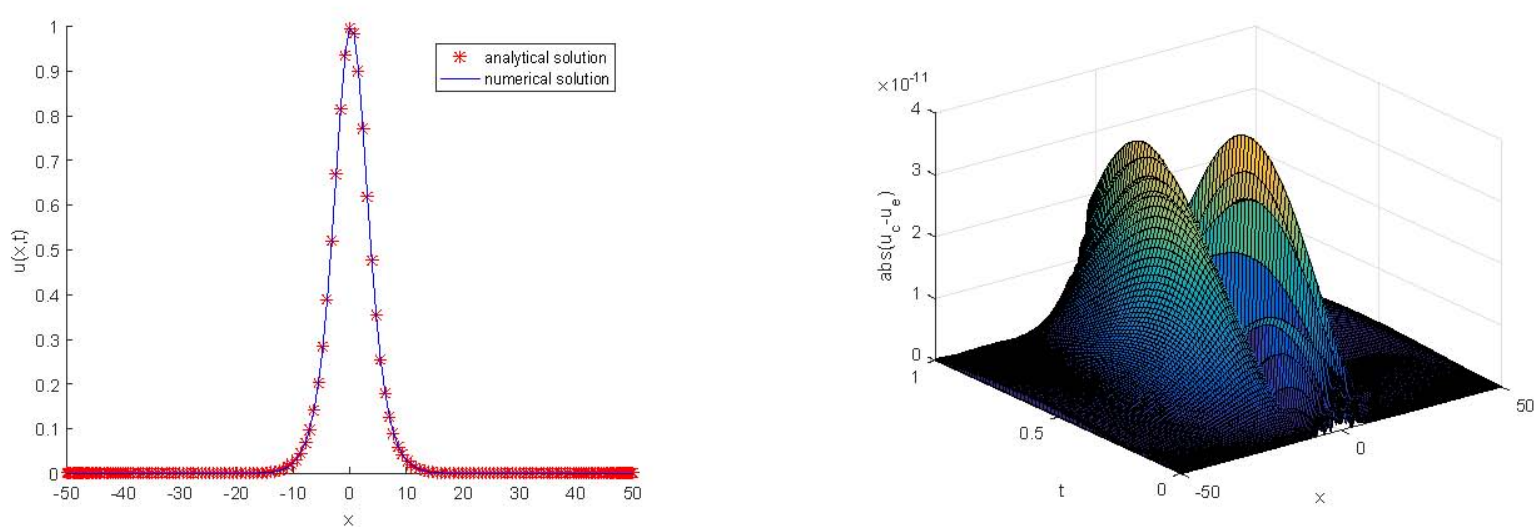

Figure 4. Single solitary wave at $t=1$ of Example 2. The absolute error of Example 2
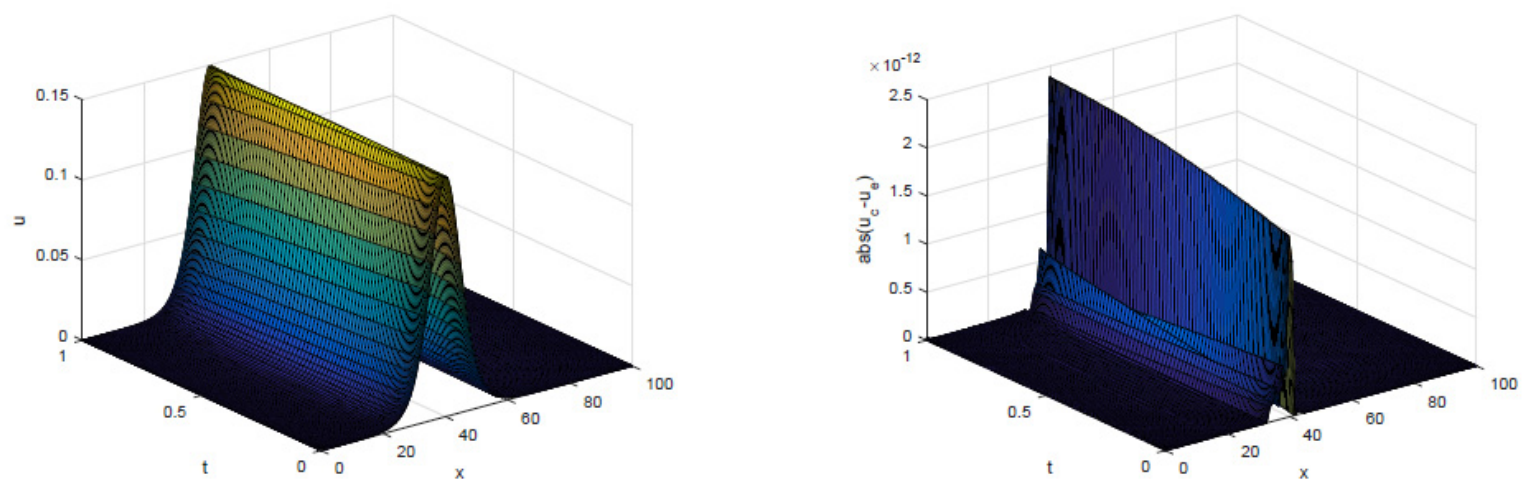

Figure 5. (Single solitary wave): The numerical solution of Example 3. The absolute error of Example 3
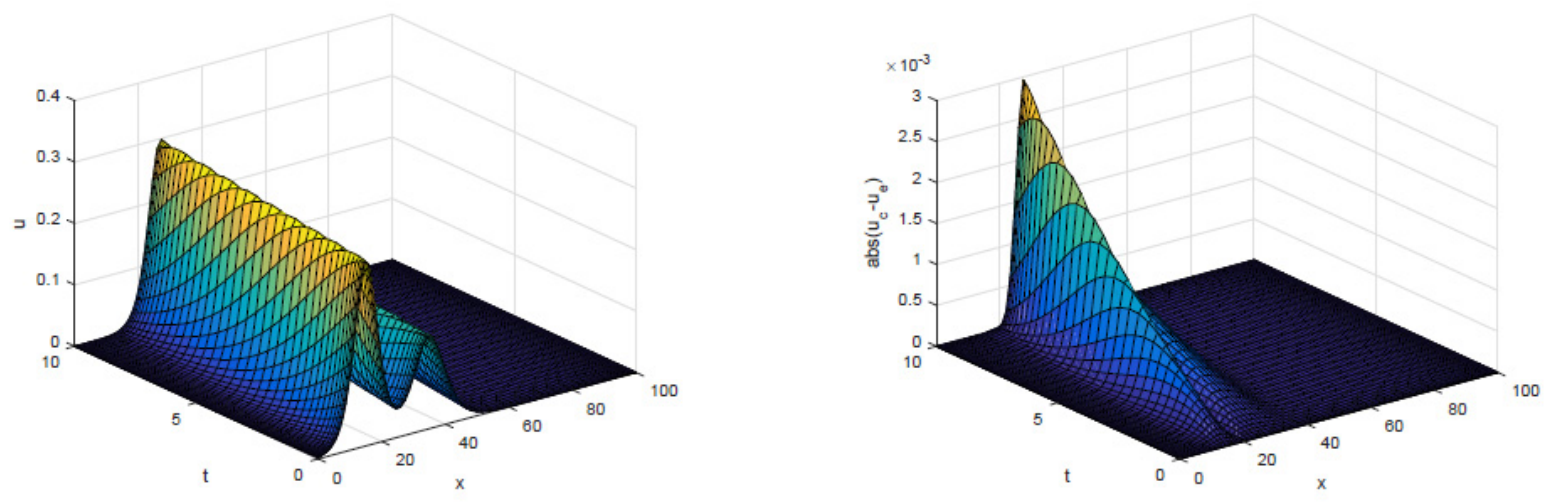

Figure 6. (Two solitary waves): The numerical solution of Example 3. The absolute error of Example 3 


\section{Discussion}

In order to solve the generalized regularized long wave equation, a new algorithm that the barycentric interpolation collocation method is constructed skillfully. Through the calculation the present method to solve the GRLW equation better. Choose the points in Examples of present method less than the other method, but the accuracy still well. Our method makes it easy to solve the GRLW equation and we can extend the present method to other the partial differential equation. The numerical results demonstrate that the new method is quite accurate and efficient for nonlinear equation. All computations are performed by the Matlab software package.

\section{Acknowledgements}

The authors would like to express their thanks to the unknown referees for their careful reading and helpful com-ments.This paper is supported by the Natural Science Foundation of China (No.11361037), the Natural Science Foundation of Inner Mongolia (No.2015MS0118).

\section{References}

Battal, G. K. S., \& Halil, Z. (2016). Solitary-wave solutions of the GRLW equationu sing septic B-spline collo-cation method. Applied Mathematics and Computation, 289(2016), 159-171.

Ben-Yu, G., \& Wei-Ming, C. (1988). The Fourier pseudo spectral method with a restrain operator for the RLW equation. Journal of Computational Physics, 74(1988), 110-126.

Djidjeli, K., Price, W. G., Twizell, E. H., \& Cao, Q. (2003). A linearized implicit pseudo-spectral method for some model equations: the regularized long wave equations. Journal for Numerical Methods in Engineering, 19(2003), 847-863.

Durn, A., \& Lpez-Marcos, M. A. (2002). Numerical behaviour of stable and unstable solitary waves. Applied Numerical Mathematics, 42(2002), 95-116.

Fu-Zheng, G., Feng, Q., \& Hong-Xing, R. (2015). Numerical simulation of the modified regularized long wave equation by split least-squares mixed finite element method. Mathematics and Computers in Simulation, 109(2015), 64-73.

Hammada, D. A., \& El-Azab, M. S. (2015). A 2N order compact finite difference method for solving the generalized regularized long wave (GRLW) equation. Applied Mathematics and Computation, 253(2015), 248-261.

Hammada, D. A., \& El-Azab, M. S. (2016). Chebyshev-Chebyshev spectral collocation method for solving the generalized regularized long wave (GRLW) equation. Applied Mathematics and Computation, 285(2016), 228-240.

Khalifa, A. K., Raslan, K. R., \& Alzubaidi, H. M. (2008). A collocation method with cubic B-splines for solving the MRLW equation. Computational and Applied Mathematics, 212(2008), 406-418.

Li-Quan, M., \& Ya-Ping, C. (2012). Numerical solutions of RLW equation using Galerkin method with extrap-olation techniques. Computer Physics Communications, 183(2012), 1609-1616.

Lu-Ming, Z. (2005). A finite difference scheme for generalized regularized long wave equation. Applied Mathematics and Computation, 168(2005), 962-972.

Maryam, M., \& Reza, M. (2011). Solving the generalized regularized long wave equation on the basis of a reproducing kernel space. Journal of Computational and Applied Mathematics, 235(2011), 4003-4014.

Noureddine, A., \& Khaled, O. (2013). Galerkin finite element method for the Rosenau-RLW equation. Comput-ers and Mathematics with Applications, 66(2013), 289-303.

Ramos, J. I. (2006). Explicit finite difference methods for the EW and RLW equations. Applied Mathematics and Computation, 179(2006), 622-638.

Raslan, K. R. (2005). A computational method for the regularized long wave (RLW) equation. Applied Math-ematics and Computation, 167(2005), 1101-1118.

Reza, M., \& Maryam M. (2010). Numerical solution of GRLW equation using Sinc-collocation method. Com-puter Physics Communications, 181(2010), 1266-1274.

Siraj-ul-Islam, Sirajul, H., \& Arshed,A. (2009). A meshfree method for the numerical solution of the RLW equation. Journal of Computational and Applied Mathematics, 223(2009), 997-1012.

Thoudam, R. (2012). A Petrov-Galerkin method for solving the generalized regularized long wave (GRLW) 
equation. Computers and Mathematics with Applications, 63(2012), 943-956.

Xin-Hui, S., Guan-Yu, X., \& Chang-Jun, L. (2013). A conservative weighted finite difference scheme for regu-larized long wave equation. Applied Mathematics and Computation, 219(2013), 9202-9209.

\section{Copyrights}

Copyright for this article is retained by the author(s), with first publication rights granted to the journal.

This is an open-access article distributed under the terms and conditions of the Creative Commons Attribution license (http://creativecommons.org/licenses/by/4.0/). 